Brief paper

\title{
Robust self-triggered control for time-varying and uncertain constrained systems via reachability analysis
}

\author{
Yulong Gao ${ }^{\text {a,* }}$, Pian Yu ${ }^{\text {a }}$, Dimos V. Dimarogonas a , Karl H. Johansson a , Lihua Xie $^{\text {b }}$ \\ a Division of Decision and Control Systems, KTH Royal Institute of Technology, SE-10044, Stockholm, Sweden \\ ${ }^{\mathrm{b}}$ School of Electrical and Electronic Engineering, Nanyang Technological University, 639798, Singapore
}

\section{A R T I C L E I N F O}

\section{Article history:}

Received 5 March 2018

Received in revised form 24 February 2019

Accepted 3 June 2019

Available online 4 July 2019

\section{Keywords:}

Constrained systems

Robust control

Self-triggered control

Reachability analysis

\begin{abstract}
A B S T R A C T
This paper develops a robust self-triggered control algorithm for time-varying and uncertain systems with constraints based on reachability analysis. The resulting piecewise constant control inputs achieve communication reduction and guarantee constraint satisfactions. In the particular case when there is no uncertainty, we propose a control design with minimum number of samplings over finite time horizon. Furthermore, when the plant is linear and the constraints are polyhedral, we prove that the previous algorithms can be reformulated as computationally tractable mixed integer linear programs. The method is compared with the robust self-triggered model predictive control in a numerical example and applied to a robot motion planning problem with temporal constraints.
\end{abstract}

(c) 2019 Elsevier Ltd. All rights reserved.

\section{Introduction}

Control for constrained systems has been extensively studied for over four decades in the literature, see e.g. Gutman and Hagander (1985) and Mayne, Rawlings, Rao, and Scokaert (2000). It is well-known that model predictive control (MPC) (Mayne et al., 2000) is a prime example of such a method. If one restricts the attention to networked control systems (NCS) subject to constraints, how to jointly design a controller and a communication protocol which can efficiently utilize network resources is a more recent challenge. In order to handle this problem, recent research efforts have been devoted to self-triggered control (Dai, Gao, Xie, Johansson, \& Xia, 2018; Heemels, Johansson, \& Tabuada, 2012), in which the next sampling time is determined in advance by the controller according to the received information.

One intuitive idea is to incorporate the self-triggered control into an MPC framework. Many results have been obtained

\footnotetext{
The work of Yulong Gao, Pian Yu, Dimos V. Dimarogonas, and Karl H. Johansson was supported in part by the Knut and Alice Wallenberg Foundation, Sweden, the Swedish Foundation for Strategic Research, and the Swedish Research Council. The work of Lihua Xie was supported by the National Natural Science Foundation of China under NSFC, China 61633014 and NSFC 61720106011 . The material in this paper was not presented at any conference. This paper was recommended for publication in revised form by Associate Editor Franco Blanchini under the direction of Editor Ian R. Petersen.

* Corresponding author.

E-mail addresses: yulongg@kth.se (Y. Gao), piany@kth.se (P. Yu), dimos@kth.se (D.V. Dimarogonas),kallej@kth.se (K.H. Johansson), elhxie@ntu.edu.sg (L. Xie).
}

for deterministic constrained systems (Barradas Berglind, Gommans, \& Heemels, 2012; Henriksson, Quevedo, Sandberg, \& Johansson, 2012). A self-triggered MPC scheme is proposed in Barradas Berglind et al. (2012) for constrained linear time-invariant systems and the inter-sampling time is maximized subject to the constraints on the cost function. For systems with uncertainty, some results of robust self-triggered MPC have been reported in Brunner, Heemels, and Allgöwer (2014, 2016). In Brunner et al. (2016), the tube MPC method is utilized to guarantee constraint satisfactions despite the presence of a bounded additive disturbance and the principle of Barradas Berglind et al. (2012) is followed to obtain the next sampling instant. In addition, the effect of the uncertainty is made use of in the design of the self-triggered mechanism.

Despite recent developments, some fundamental issues remain for self-triggered MPC. The incorporation of the self-triggered scheme into MPC does not immediately preserve the conventional recursive feasibility and closed-loop stability of MPC. In order to guarantee these two properties, the price of more computational effort at the sampling instants is paid to satisfy the constraints on the cost function when maximizing the inter-sampling time.

Other than MPC, only a few work address the self-triggered control for constrained systems. For example, the focus of Lehmann, Kiener, and Johansson (2012), Seuret, Prieur, Tarbouriech, and Zaccarian (2013) and Wu, Reimann, and Liu (2014) is on the design of an event-triggered controller when the systems are subject to actuator saturation. One recent work (Hashimoto, Adachi, \& Dimarogonas, 2018) provides a contractive set-based approach to design self-triggered control for linear deterministic constrained systems. 
Different from the above results, this paper aims at proposing a robust self-triggered control framework for time-varying and uncertain systems with constraints. To the best of our knowledge, this topic has not been explored up to now and cannot be handled by the previous mentioned methods, such as self-triggered MPC. The main challenges are: (1) how to guarantee recursive feasibility in a time-varying setup by self-triggered control; (2) how to ensure constraint satisfaction for any disturbance realization. In this work, we make full use of reachability analysis to handle these issues. Although reachability has been widely studied (Bertsekas \& Rhodes, 1971; Lygeros, Tomlin, \& Sastry, 1999; Raković, Kerrigan, Mayne, \& Lygeros, 2006), the incorporation of reachability into self-triggered control is novel. One recent work (AlKhatib, Girard, \& Dang, 2017) uses reachability-based self-triggered control to design the variable sampling period for sampled-data linear systems. However, neither constraints nor uncertainties are considered in AlKhatib et al. (2017).

The use of reachability analysis in this paper provides a geometric interpretation for the self-triggered control from a set theoretical point of view. Available geometry software tools facilitate the implementation of our algorithms. Some practical applications of our algorithm include control of hybrid systems and robot motion planning (see Example 2). The main contributions are summarized below.

- We propose a novel robust self-triggered control algorithm (Algorithm 1) for time-varying and uncertain systems with constraints. Constraint satisfactions and recursive feasibility are shown to be guaranteed based on reachability analysis. We calculate the maximum inter-sampling time by solving the corresponding optimization problem $\left(\mathcal{P}_{\left[k_{i}, N\right]}^{1}\left(x_{k_{i}}\right)\right)$ only once at each sampling instant, which avoids the repetitive computation required in the self-triggered MPC. In the particular case when there is no uncertainty, we develop a control method with minimum number of samplings over a finite time horizon. This is achieved by solving the optimization problem $\left(\mathcal{P}_{[0, N]}^{2}\left(x_{0}\right)\right)$ only once.

- When the plant is linear and the constraints are polyhedral, we prove that all the optimization problems $\left(\mathcal{P}_{\left[k_{i}, N\right]}^{1}\left(x_{k_{i}}\right)\right.$ and $\left.\mathcal{P}_{[0, N]}^{2}\left(x_{0}\right)\right)$ can be reformulated as mixed integer linear programming (MILP) problems, which are, in our cases, computationally tractable (Theorems 4.1 and 4.2 ). The numerical comparisons (Example 1) show that our algorithm achieves a better communication reduction and faster online computation than the robust self-triggered MPC in Brunner et al. (2014) without much loss in performance.

The remainder of the paper is organized as follows. The problem statement is given in Section 2. Section 3 presents the robust self-triggered control algorithm. The specialization to linear plants with polyhedral constraints is provided in Section 4. Two examples in Section 5 illustrate the effectiveness of our approach. Finally, Section 6 concludes this paper.

Notation. Let $\mathbb{N}$ be the set of nonnegative integers. For some $q, s \in \mathbb{N}$ and $q<s$, let $\mathbb{N}_{[q, s]}$ denote the set $\{r \in \mathbb{N} \mid q \leq r \leq$ $s\}$, respectively. When $\leq, \geq,<$, and $>$, are applied to vectors, they are interpreted element-wise. A column vector of ones with appropriate dimension is denoted by $\mathbf{1}$. The Minkowski sum of two sets is denoted by $\mathbb{A} \oplus \mathbb{B}=\{a+b \mid \forall a \in \mathbb{A}, \forall b \in \mathbb{B}\}$. The Minkowski difference of two sets is denoted by $\mathbb{A} \ominus \mathbb{B}=$ $\{c \mid \forall b \in \mathbb{B}, c+b \in \mathbb{A}\}$. For a vector $x \in \mathbb{R}^{n}$, define $\|x\|_{\infty}=$ $\max _{i}\left|x_{i}\right|$. For a polyhedron $\mathbb{P}=\left\{x \in \mathbb{R}^{n} \mid P x \leq p\right\}$, define $\|\mathbb{P}\|_{\infty}=\max _{x \in \mathbb{P}}\left\{\|P x-p\|_{\infty}\right\}$. For $\mathbb{X} \subseteq \mathbb{R}^{n}$ and $A \in \mathbb{R}^{n \times n}$, define $A^{-1} \mathbb{X}=\left\{x \in \mathbb{R}^{n} \mid A x \in \mathbb{X}\right\}$. For $x_{l} \in \mathbb{R}^{n}, l \in \mathbb{N}$, define $\sum_{l=k}^{j} x_{l}=\mathbf{0}$ if $k>j$. For $\mathbb{X}_{l} \subseteq \mathbb{R}^{n}$ and $A_{l} \in \mathbb{R}^{n \times n}, l \in \mathbb{N}$, define

$\bigoplus_{l=k}^{j} \mathbb{X}_{l}=\left\{\begin{array}{l}\emptyset, k>j, \\ \mathbb{X}_{k} \oplus \mathbb{X}_{k+1} \oplus \ldots \mathbb{X}_{j}, \quad k \leq j,\end{array}\right.$ $\prod_{l=k}^{j} A_{l}=\left\{\begin{array}{l}I, k>j, \\ A_{j} A_{j-1} \ldots A_{k}, k \leq j .\end{array}\right.$

Given two sets $\mathbb{X}$ and $\tilde{\mathbb{X}}$, define

$\mathbb{1}_{\mathbb{X}}(x)=\left\{\begin{array}{l}1, x \in \mathbb{X}, \\ 0, x \notin \mathbb{X},\end{array} \quad\right.$ and $\mathbb{1}_{\mathbb{X}}(\tilde{\mathbb{X}})= \begin{cases}1, & \tilde{\mathbb{X}} \subseteq \mathbb{X}, \\ 0, & \tilde{\mathbb{X}} \nsubseteq \mathbb{X} .\end{cases}$

\section{Problem statement}

Consider a discrete-time dynamic control system

$x_{k+1}=f_{k}\left(x_{k}, u_{k}\right)+w_{k}$,

where $x_{k} \in \mathbb{R}^{n_{x}}$ and $u_{k} \in \mathbb{R}^{n_{u}}, w_{k} \in \mathbb{R}^{n_{x}}$, and $f_{k}: \mathbb{R}^{n_{x}} \times \mathbb{R}^{n_{u}} \rightarrow \mathbb{R}^{n_{x}}$. The control input $u_{k}$ at time $k$ is constrained by a set $\mathbb{U}_{k} \subset$ $\mathbb{R}^{n_{u}}$. The additive disturbance $w_{k}$ at time instant $k$ belongs to a compact set $\mathbb{W}_{k} \subset \mathbb{R}^{n_{x}}$. The initial state $x_{0}$ is contained in a given set $\mathbb{X}_{0} \subset \mathbb{R}^{n_{x}}$. In addition, given a finite time horizon $N \in \mathbb{N}$, the system (1) is subject to a target tube, denoted by $\left\{\left(\mathbb{X}_{k}, k\right), k \in \mathbb{N}_{[1, N]}\right\}$, where $\mathbb{X}_{k} \subseteq \mathbb{R}^{n_{x}}, \forall k \in \mathbb{N}_{[1, N]}$. It is assumed that the function $f_{k}$ and the disturbance set $\mathbb{W}_{k}$ are known for all $k \in \mathbb{N}_{[0, N-1]}$.

Assumption 2.1. The function $f_{k}(x, u), \forall k \in \mathbb{N}_{[0, N-1]}$, is continuous in $x$ and $u$, respectively.

Assumption 2.2. The sets $\mathbb{U}_{k}, \forall k \in \mathbb{N}_{[0, N-1]}$, and $\mathbb{X}_{k}, \forall k \in \mathbb{N}_{[0, N]}$, are compact.

The objective of this paper is to develop a self-triggered control algorithm for the system (1), thereby yielding a sequence of piecewise constant control inputs. More specifically, we aim to determine a sequence of sampling instants $\left\{k_{0}, k_{1}, \ldots, k_{T}\right\}$ with $k_{0}=0, k_{i+1}=k_{i}+M_{i}$, and $k_{T}=N$ such that $u_{j}=u_{l}, \forall j, l \in$ $\mathbb{N}_{\left[k_{i}, k_{i+1}-1\right]}$ and all the constraints are satisfied at each time instant $k \in \mathbb{N}_{[0, N]}$. Here, $T+1$ is the total number of samplings within $N$ time instants, which quantifies the communication consumption, and $M_{i}$ denotes the inter-sampling time between $k_{i}$ and $k_{i+1}$.

\section{Self-triggered control for constrained systems via reacha- bility analysis}

In this section, we will provide a reachability-based selftriggered control algorithm for the uncertain constrained system (1). Furthermore, a control method with minimum number of samplings will be designed when the system (1) is reduced to be deterministic, i.e., $\mathbb{W}_{k}=\{0\}$.

\subsection{Robust self-triggered control}

\subsubsection{Computation of reachable sets}

Definition 3.1. The target tube $\left\{\left(\mathbb{X}_{k}, k\right), k \in \mathbb{N}_{[1, N]}\right\}$ of the system (1) is reachable from the initial state $x_{0} \in \mathbb{X}_{0}$ if there exists a sequence of control inputs $u_{k} \in \mathbb{U}_{k}, \forall k \in \mathbb{N}_{[0, N-1]}$, such that the state $x_{k} \in \mathbb{X}_{k}, \forall k \in \mathbb{N}_{[1, N]}$, for all possible disturbance sequences $w_{k} \in \mathbb{W}_{k}, \forall k \in \mathbb{N}_{[0, N-1]}$.

Let $\mathbb{X}_{N}^{*}=\mathbb{X}_{N}$. For $k \in \mathbb{N}_{[0, N-1]}$, the backward reachable set $\mathbb{X}_{k}^{*}$ for the system (1) is recursively computed by:

$\mathbb{P}_{k}=\left\{z \in \mathbb{R}^{n_{x}} \mid \exists u_{k} \in \mathbb{U}_{k}, f_{k}\left(z, u_{k}\right) \oplus \mathbb{W}_{k} \subseteq \mathbb{X}_{k+1}^{*}\right\}$,

$\mathbb{X}_{k}^{*}=\mathbb{P}_{k} \cap \mathbb{X}_{k}$.

Proposition 3.1 (Bertsekas $\mathcal{E}$ Rhodes, 1971). The target tube $\left\{\left(\mathbb{X}_{j}, j\right)\right.$, $\left.j \in \mathbb{N}_{[k+1, N]}\right\}$ of the system (1) is reachable from $x_{k}$ if and only if $x_{k} \in \mathbb{P}_{k}$. Furthermore, the target tube $\left\{\left(\mathbb{X}_{k}, k\right), k \in \mathbb{N}_{[1, N]}\right\}$ is reachable from the initial state $x_{0} \in \mathbb{X}_{0}$ if and only if $x_{0} \in \mathbb{X}_{0}^{*}$. 
According to Raković et al. (2006), Assumptions 2.1 and 2.2 make the resulting reachable sets compact. Proposition 3.1 indicates that if the state $x_{j} \in \mathbb{X}_{j}^{*}$, the recursive feasibility and the constraint satisfactions can be guaranteed.

Remark 3.1. There exist some methods to compute the reachable sets for a nonlinear system (1), e.g., Chen, Herbert, Vashishtha, Bansal, and Tomlin (2018) and Raković et al. (2006). In addition, there are results on the inner approximations of the reachable sets $\mathbb{X}_{k}^{*}$, e.g., Althoff and Krogh (2014) and Mitchell (2011). Note that these inner approximations are applicable also for the following algorithms, since they provide constraint satisfaction and recursive feasibility guarantees.

Remark 3.2. Given the initial state $x_{0}$, one can choose the minimal horizon $N$ such that $\left\{\left(\mathbb{X}_{j}, j\right), j \in \mathbb{N}_{[0, N]}\right\}$ is reachable from $x_{0}$.

\subsubsection{Algorithm}

Define the self-triggered condition for the system (1) as

$$
\begin{gathered}
k_{i+1}=\max \left\{k \mid k_{i}<k \leq N \text { such that } u_{j}=u \in \mathbb{U}_{j},\right. \\
j \in \mathbb{N}_{\left[k_{i}, k-1\right]}, \text { and the target tube } \\
\left.\left\{\left(\mathbb{X}_{j}, j\right), j \in \mathbb{N}_{\left[k_{i}, N\right]}\right\} \text { of }(1) \text { is reachable }\right\} .
\end{gathered}
$$

Recall that $k_{i+1}=k_{i}+M_{i}$. The following lemma provides the formulation to compute $M_{i}$.

Proposition 3.2. Given the state $x_{k_{i}} \in \mathbb{X}_{k_{i}}^{*}, k_{i} \in \mathbb{N}_{[0, N-1]}$, the intersampling time $M_{i}$ is obtained by solving the following optimization problem, denoted by $\mathcal{P}_{\left[k_{i}, N\right]}^{1}\left(x_{k_{i}}\right)$ :

$$
\max _{u} \sum_{j=k_{i}+1}^{N} r_{j}
$$

subject to

$$
\begin{gathered}
\tilde{\mathbb{X}}_{\left[k_{i}, k_{i}\right]}=\left\{x_{k_{i}}\right\}, \\
\forall j \in \mathbb{N}_{\left[k_{i}, N-1\right]}: \tilde{\mathbb{X}}_{\left[k_{i}, j+1\right]}=f_{j}\left(\tilde{\mathbb{X}}_{\left[k_{i}, j\right]}, u\right) \oplus \mathbb{W}_{j}, \\
\forall j \in \mathbb{N}_{\left[k_{i}+1, N\right]}: \\
r_{j}=\left\{\begin{array}{l}
\mathbb{1}_{\mathbb{X}_{k_{i}+1}^{*}}\left(\tilde{\mathbb{X}}_{\left[k_{i}, k_{i}+1\right]}\right) \mathbb{1}_{\mathbb{U}_{k_{i}}}(u), j=k_{i}+1, \\
r_{j-1} \mathbb{1}_{\mathbb{X}_{j}^{*}}\left(\tilde{\mathbb{X}}_{\left[k_{i}, j\right]}\right) \mathbb{1}_{\mathbb{U}_{j-1}}(u), j>k_{i}+1,
\end{array}\right.
\end{gathered}
$$

where $f_{j}(\mathbb{X}, u)=\left\{z \in \mathbb{R}^{n_{x}} \mid z=f(x, u), \forall x \in \mathbb{X}\right\}$. That is, $M_{i}=\sum_{j=k_{i}+1}^{N} r_{j}^{*}$, where $r_{j}^{*}$ corresponds to the optimal solution of $\mathcal{P}_{\left[k_{i}, N\right]}^{1}\left(x_{k_{i}}\right)$.

Proof. The definition of $r_{j}$ characterizes the successive constraint satisfactions from time $k_{i}$ for all possible disturbances $w_{l} \in \mathbb{W}_{l}, \forall l \in \mathbb{N}_{\left[k_{i}, j-1\right]}$. Then, the proof directly follows from Proposition 3.1 and the objective function of $\mathcal{P}_{\left[k_{i}, N\right]}^{1}\left(x_{k_{i}}\right)$.

The geometric interpretation of the optimization problem $\mathcal{P}_{\left[k_{i}, N\right]}^{1}\left(x_{k_{i}}\right)$ is to seek a fixed control input $u$ such that starting from time $k_{i}$, the time length, during which the state constraints and the control input constraints are satisfied for all possible disturbances, is maximized.

We denote by $u^{*}$ the optimal solution to the optimization problem $\mathcal{P}_{\left[k_{i}, N\right]}^{1}\left(x_{k_{i}}\right)$. The following Algorithm 1 presents the robust self-triggered control for the uncertain constrained system (1).

\subsection{Control with minimum number of samplings}

This subsection will provide a control method with minimum number of samplings, denoted by $T^{*}+1$, over a given finite

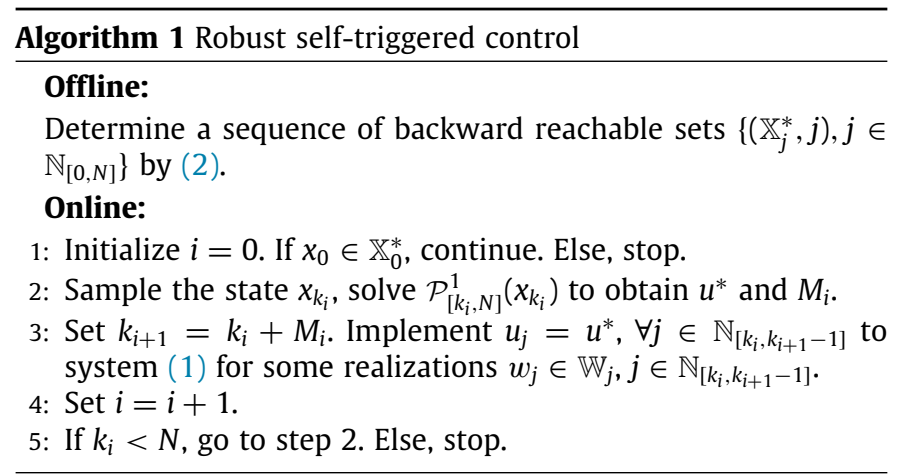

horizon for the system (1). Without loss of generality, we assume that $N \geq 2$.

Proposition 3.3. The minimum number of samplings $T^{*}+1$ is obtained by solving the following optimization problem, denoted by $\mathcal{P}_{[0, N]}^{2}\left(x_{0}\right)$,

$\min _{u_{0}, \Delta_{j}} \sum_{j=0}^{N-2}\left(1-\mathbb{1}_{\{0\}}\left(\Delta_{j}\right)\right)$

subject to

$\forall j \in \mathbb{N}_{[0, N-1]}: x_{j+1}=f_{j}\left(x_{j}, u_{j}\right)$,

$\forall j \in \mathbb{N}_{[0, N-1]}: u_{j}=\left\{\begin{array}{l}u_{0}, j=0 \\ u_{j-1}+\Delta_{j-1}, \quad j \geq 1,\end{array}\right.$

$\forall j \in \mathbb{N}_{[1, N]}: x_{j} \in \mathbb{X}_{j}^{*}$

$\forall j \in \mathbb{N}_{[0, N-1]}: u_{j} \in \mathbb{U}_{j}$.

That is, $T^{*}=\sum_{j=0}^{N-2}\left(1-\mathbb{1}_{\{0\}}\left(\Delta_{j}^{*}\right)\right)$, where $\Delta_{j}^{*}$ corresponds to the optimal solution of $\mathcal{P}_{[0, N]}^{2}\left(x_{0}\right)$.

Proof. In (4c), $\Delta_{j-1}$ denotes the difference between $u_{j}$ and $u_{j-1}$. The objective function of $\mathcal{P}_{[0, N]}^{2}\left(x_{0}\right)$ aims at maximizing the number of zero (i.e., $\Delta_{j}=0$ ) over the time interval $\mathbb{N}_{[0, N-1]}$. Thus, the optimal solution generates a sequence of piecewise constant control inputs with minimum number of switching times.

Note that Algorithm 1 is applicable for deterministic systems. In this case, the difference is that Algorithm 1 cannot guarantee the achievement of minimum number of samplings for deterministic systems.

Remark 3.3. For uncertain constrained systems, it is difficult to design the control with minimum number of samplings since at each sampling instant $k_{i}$, the exact executions of the future disturbance $w_{j}, \forall j \in \mathbb{N}_{\left[k_{i}, N-1\right]}$, are unknown.

\section{Self-triggered control for linear systems with polyhedral constraints}

The development of geometry software allows us to compute the sets $\mathbb{X}_{k}^{*}$ exactly and efficiently if the system is linear and the constraint sets are polyhedral (Herceg, Kvasnica, Jones, \& Morari, 2013). This section will specialize the systems (1) to be linear and the constraint sets to be polyhedral. We can reformulate the optimization problems $\mathcal{P}_{\left[k_{i}, N\right]}^{1}\left(x_{k_{i}}\right)$ and $\mathcal{P}_{[0, N]}^{2}\left(x_{0}\right)$ to be computationally tractable MILP problems.

If the model $f_{k}$ is linear, the system (1) becomes

$x_{k+1}=A_{k} x_{k}+B_{k} u_{k}+w_{k}$. 
Here $A_{k}$ and $B_{k}$ are deterministic real matrices with appropriate dimensions at each time $k \in \mathbb{N}_{[0, N-1]}$. The control input sets $\mathbb{U}_{k}$, $k \in \mathbb{N}_{[0, N-1]}$, are compact polyhedra. Each set $\mathbb{X}_{k}$ of the target tube $\left\{\left(\mathbb{X}_{k}, k\right), k \in \mathbb{N}_{[1, N]}\right\}$ is a compact polyhedron. The disturbance sets $\mathbb{W}_{k}, k \in \mathbb{N}_{[0, N-1]}$, are compact polyhedra.

Now, the computation of the sets $\mathbb{X}_{k}^{*}$ in (2) is given as follows. Note that the following equations involve only set operations and the corresponding sets can be well-defined even if the matrix $A_{k}$ is not invertible. Hence, we do not impose any assumption on $A_{k}$.

Lemma 4.1 (Bertsekas \& Rhodes, 1971). For the uncertain linear system (5) with polyhedral constraints, the set $\mathbb{X}_{k}^{*}$ in (2) evolves as

$\mathbb{Q}_{k}=\mathbb{X}_{k}^{*} \ominus \mathbb{W}_{k}$

$\mathbb{P}_{k}=A_{k}^{-1}\left(\mathbb{Q}_{k+1} \oplus\left(-B_{k} \mathbb{U}_{k}\right)\right)$,

$\mathbb{X}_{k}^{*}=\mathbb{P}_{k} \cap \mathbb{X}_{k}, \mathbb{X}_{N}^{*}=\mathbb{X}_{N}$.

Remark 4.1. Since the sets $\mathbb{X}_{k}, k \in \mathbb{N}_{[0, N]}$, are compact, the sets $\mathbb{X}_{k}^{*}, k \in \mathbb{N}_{[0, N]}$, are also compact even when the matrices $A_{k}$ are not invertible.

The polyhedral sets $\mathbb{U}_{k}$ and $\mathbb{X}_{k}^{*}$ in (6) are written as

$\mathbb{U}_{k}=\left\{z \in \mathbb{R}^{n_{u}} \mid E_{k} z \leq e_{k}\right\}$,

$\mathbb{X}_{k}^{*}=\left\{z \in \mathbb{R}^{n_{x}} \mid F_{k} z \leq f_{k}\right\}$,

where $E_{k}$ and $F_{k}$ (or $e_{k}$ and $f_{k}$ ) are matrices (or vectors) with appropriate dimensions.

\subsection{Robust self-triggered control}

Before providing the main result, we need some preliminary lemmas.

Lemma 4.2. The set $\tilde{\mathbb{X}}_{\left[k_{i}, j\right]}$ in (3c) can be written as

$\tilde{\mathbb{X}}_{\left[k_{i}, j\right]}=\left(G_{\left[k_{i}, j\right]} x_{k}+H_{\left[k_{i}, j\right]} u\right) \oplus \mathbb{Z}_{\left[k_{i}, j\right]}, j \in \mathbb{N}_{\left[k_{i}, N\right]}$,

where $G_{\left[k_{i}, j\right]}=\prod_{l=k_{i}}^{j-1} A_{l}, H_{\left[k_{i}, j\right]}=\sum_{m=k_{i}}^{j-1} \prod_{l=m+1}^{j-1} A_{l} B_{m}, \mathbb{Z}_{\left[k_{i}, j\right]}=$ $\bigoplus_{m=k_{i}}^{j-1} \prod_{l=m+1}^{j-1} A_{l} \mathbb{W}_{m}$. Furthermore, the set $\mathbb{Z}_{\left[k_{i}, j\right]}$ is a closed polyhedron.

Proof. When $j=k_{i}$, (7) implies that $\tilde{\mathbb{X}}_{\left[k_{i}, k_{i}\right]}=\left\{x_{k_{i}}\right\}$. According to the definition of $\tilde{\mathbb{X}}_{\left[k_{i}, j\right]}, j \in \mathbb{N}_{\left[k_{i}+1, N\right]}$, in (3c), by induction, it follows

$$
\begin{aligned}
& \tilde{\mathbb{X}}_{\left[k_{i}, j+1\right]} \\
= & A_{j} \tilde{\mathbb{X}}_{\left[k_{i}, j\right]} \oplus B_{j} u \oplus \mathbb{W}_{j} \\
= & A_{j}\left(G_{\left[k_{i}, j\right]} x_{k_{i}}+H_{\left[k_{i}, j\right]} u \oplus \mathbb{Z}_{\left[k_{i}, j\right]}\right) \oplus B_{j} u \oplus \mathbb{W}_{j} \\
= & \left(A_{j} \prod_{l=k_{i}}^{j-1} A_{l} x_{k_{i}}+\left(A_{j} \sum_{m=k_{i}}^{j-1} \prod_{l=m+1}^{j-1} A_{l} B_{m}+B_{j}\right) u\right) \\
& \oplus\left(A_{j} \bigoplus_{m=k_{i}}^{j-1} \prod_{l=m+1}^{j-1} A_{l} \mathbb{W}_{m} \oplus \mathbb{W}_{j}\right) \\
= & \left(\prod_{l=k_{i}}^{j} A_{l} x_{k_{i}}+\sum_{m=k_{i}}^{j} \prod_{l=m+1}^{j} A_{l} B_{m}\right) \oplus\left(\bigoplus_{m=k_{i}}^{j} \prod_{l=m+1}^{j} A_{l} \mathbb{W}_{m}\right) \\
= & \left(G_{\left[k_{i}, j+1\right]} x_{k_{i}}+H_{\left[k_{i}, j+1\right]} u\right) \oplus \mathbb{Z}_{\left[k_{i}, j+1\right]} .
\end{aligned}
$$

Since the sets $\mathbb{W}_{m}$ are compact polyhedra, we have that the sets $\mathbb{Z}_{\left[k_{i}, j\right]}$ are closed polyhedra.

Lemma 4.3 (Blanchini \& Miani, 2007). Given two polyhedra $\mathbb{P}=$ $\left\{z \in \mathbb{R}^{n} \mid P z \leq p\right\}$ and $\mathbb{Q}=\left\{z \in \mathbb{R}^{n} \mid Q z \leq q\right\}, \mathbb{P} \subseteq \mathbb{Q}$ holds if and only if there exists a non-negative matrix $S$ such that $S P=Q$ and $S p \leq q$.
Assume now that $\mathbb{Z}_{\left[k_{i}, j\right]}=\left\{z \in \mathbb{R}^{n_{x}} \mid V_{\left[k_{i}, j\right]} z \leq v_{\left[k_{i}, j\right]}\right\}$, $j \in \mathbb{N}_{\left[k_{i}+1, N\right]}$, where the matrix $V_{\left[k_{i}, j\right]}$ and vector $v_{\left[k_{i}, j\right]}$ can be computed offline according to $\mathbb{Z}_{\left[k_{i}, j\right]}=\bigoplus_{m=k_{i}}^{j-1} \prod_{l=m+1}^{j-1} A_{l} \mathbb{W}_{m}$. By Lemma 4.3, we derive the following result.

Lemma 4.4. For the sets $\tilde{\mathbb{X}}_{\left[k_{i}, j\right]}$ and $\mathbb{X}_{j}^{*}, \tilde{\mathbb{X}}_{\left[k_{i}, j\right]} \subseteq \mathbb{X}_{j}^{*}$ holds if and only if there exists a non-negative matrix $S_{\left[k_{i}, j\right]}$ such that

$S_{\left[k_{i}, j\right]} V_{\left[k_{i}, j\right]}=F_{j}$,

$S_{\left[k_{i}, j\right]}\left(v_{\left[k_{i}, j\right]}+V_{\left[k_{i}, j\right]}\left(G_{\left[k_{i}, j\right]} x_{k_{i}}+H_{\left[k_{i}, j\right]} u\right)\right) \leq f_{j}$.

Proof. This directly follows from Lemmas 4.2-4.3.

Since $u$ is the decision variable, the constraints (9) are nonlinear. To remedy this, we can calculate the nonnegative matrices $S_{\left[k_{i}, j\right]}$ offline to satisfy (8) by an LP (Fleming, Kouvaritakis, \& Cannon, 2013):

$\left(S_{\left[k_{i}, j\right]}\right)_{l}=\underset{a^{T}}{\operatorname{argmin}}\left\{\mathbf{1}^{T} a \mid a^{T} V_{\left[k_{i}, j\right]}=\left(F_{j}\right)_{l}, a \geq \mathbf{0}\right\}$,

where $a$ is a vector with appropriate dimension and $(S)_{l}$ denotes the lth row of the matrix $S$.

Remark 4.2. The LP in (10) admits a nonnegative matrix solution with minimum infinity norm, which could lead to a larger feasible region for the optimization problem $\mathcal{P}_{\left[k_{i}, N\right]}^{1}\left(x_{k_{i}}\right)$ than other nonnegative solutions to (8).

The next theorem shows that the robust self-triggered control for the system (5) with polyhedral constraints can be designed by solving a computationally tractable MILP.

Theorem 4.1. For the uncertain linear system (5) with polyhedral constraints, the problem $\mathcal{P}_{\left[k_{i}, N\right]}^{1}\left(x_{k_{i}}\right)$ can be reformulated as an MILP, denoted by $\mathcal{P}_{\left[k_{i}, N\right]}^{3}\left(x_{k_{i}}\right)$,

$\max _{u, \delta_{j}} \sum_{j=k_{i}+1}^{N}\left(1-\delta_{j}\right)$

subject to

$\forall j \in \mathbb{N}_{\left[k_{i}+1, N\right]}$ :

$$
S_{\left[k_{i}, j\right]}\left(\tilde{G}_{\left[k_{i}, j\right]} x_{k_{i}}+\tilde{H}_{\left[k_{i}, j\right]} u\right) \leq \tilde{f}_{\left[k_{i}, j\right]}+\delta_{j} \Gamma \mathbf{1},
$$

$\forall j \in \mathbb{N}_{\left[k_{i}, N-1\right]}: E_{j} u \leq e_{j}+\delta_{j} \Gamma \mathbf{1}$,

$\forall j \in \mathbb{N}_{\left[k_{i}+1, N-1\right]}: \delta_{j} \leq \delta_{j+1}$,

$\forall j \in \mathbb{N}_{\left[k_{i}+1, N\right]}: \delta_{j} \in\{0,1\}$,

where $\tilde{G}_{\left[k_{i}, j\right]}=V_{\left[k_{i}, j\right]} G_{\left[k_{i}, j\right]}, \tilde{H}_{\left[k_{i}, j\right]}=V_{\left[k_{i}, j\right]} H_{\left[k_{i}, j\right]}, \tilde{f}_{\left[k_{i}, j\right]}=f_{j}-S_{\left[k_{i}, j\right]} v_{\left[k_{i}, j\right]}$, and $\Gamma$ is a positive constant satisfying

$$
\begin{aligned}
& \Gamma>\max \left\{\max _{j \in \mathbb{N}_{\left[k_{i}, N-1\right]}}\left\|\mathbb{U}_{j}\right\|_{\infty},\right. \\
& \left.\max _{j \in \mathbb{N}_{\left[k_{i}+1, N\right]}} \max _{u \in \mathbb{U}_{j}}\left\|S_{\left[k_{i}, j\right]}\left(\tilde{G}_{\left[k_{i}, j\right]} x_{k_{i}}+\tilde{H}_{\left[k_{i}, j\right]} u\right)-\tilde{f}_{\left[k_{i}, j\right]}\right\|_{\infty}\right\} .
\end{aligned}
$$

Proof. Recall the definition of $r_{j}$ in (3d). Let us introduce a sequence of $0-1$ variables $\delta_{j}, j \in \mathbb{N}_{\left[k_{i}+1, N\right]}$. By setting $r_{j}=1-\delta_{j}$, we have

$$
\left\{\begin{aligned}
& \forall l \in \mathbb{N}_{\left[k_{i}+1, j\right]}: \\
& S_{\left[k_{i}, l\right]}\left(\tilde{G}_{\left[k_{i}, l\right]} x_{k_{i}}+\tilde{H}_{\left[k_{i}, l\right]} u\right) \leq \tilde{f}_{\left[k_{i}, l\right]}+\delta_{l} \Gamma \mathbf{1}, \\
& \forall l \in \mathbb{N}_{\left[k_{i}, j-1\right]}: E_{l} u \leq e_{l}+\delta_{l} \Gamma \mathbf{1},
\end{aligned}\right.
$$

where $\Gamma$ is a positive number satisfying (12). Furthermore, $\forall j \in$ $\mathbb{N}_{\left[k_{i}+1, N-1\right]}, r_{j} \geq r_{j+1}$ can be rewritten as $\delta_{j} \leq \delta_{j+1}$. Then, we get the problem $\mathcal{P}_{\left[k_{i}, N\right]}^{2}\left(x_{k_{i}}\right)$. 
Remark 4.3. The computational complexity of the MILP (11) can be analyzed as follows. Considering the constraint (11d) on $\delta_{j}$, it is easy to construct an enumeration tree with at most $N-k_{i}$ nodes, each of which is in the form of $\delta_{j}=0, \forall j \in$ $\mathbb{N}_{\left[k_{i}+1, k\right]}$, and $\delta_{j}=1, \forall j \in \mathbb{N}_{[k+1, N]}$, for some $k \in \mathbb{N}_{\left[k_{i}+1, N\right]}$. This construction avoids the combinatorial explosion issue. By fixing the enumeration sequence $\left\{\delta_{j}\right\}_{k_{i}+1}^{N}$, the constraints (11b)-(11c) become linear in the decision variable $u$ and in particular are redundant for $j \in \mathbb{N}_{[k+1, N]}$. Hence, the MILP (11) can be solved through several LPs by increasing $k$ until the LP is infeasible. The computational complexity of the original MILP (11) is at most $N-k_{i}$ times the computational complexity of the LP. Note that the number of constraints in the LP is at most the total number of the inequalities in (11b)-(11c) over $j \in \mathbb{N}_{[0, N]}$, denoted by $L$. From Megiddo (1984), we conclude that the problem (11) can be solved in $\mathcal{O}(N L)$ time. In addition, several software tools have been developed to solve large MILPs in the past few years, e.g., Linderoth and Lodi (2010), also allowing us to solve our problem online efficiently.

Remark 4.4. Following similar operations as for the linear case, the previous optimization problem $\mathcal{P}_{\left[k_{i}, N\right]}^{1}\left(x_{k_{i}}\right)$ can be reformulated as an integer program with a constraint like (11d). The resulting integer program can be iteratively cast as a classic constrained robust nonlinear control problem.

Remark 4.5. In general, $\Gamma$ can be arbitrarily chosen to be a sufficiently large positive constant. The lower bound on $\Gamma$ defined in (12) aims at quantifying how large $\Gamma$ should be, which can be calculated by solving a linear program (LP) since the sets $\mathbb{U}_{j}$ are compact polyhedra and the norm is the $\ell_{\infty}-$ norm.

\subsection{Control with minimum number of samplings}

When the disturbance set $\mathbb{W}_{k}=\{0\}, \forall k \in \mathbb{N}_{[0, N-1]}$, we can also reformulate the optimization problem $\mathcal{P}_{[0, N]}^{2}\left(x_{0}\right)$ as a computationally tractable MILP.

Theorem 4.2. For the deterministic linear system (5) with polyhedral constraints, the problem $\mathcal{P}_{[0, N]}^{2}\left(x_{0}\right)$ can be reformulated as an MILP, denoted by $\mathcal{P}_{[0, N]}^{4}\left(x_{0}\right)$,

$\max _{u_{0}, \Delta_{j}, \delta_{j}, c_{j}} J_{[0, N]}\left(x_{0}\right)=\sum_{j=0}^{N-2}\left(1-\delta_{j}\right)$

subject to

$\forall j \in \mathbb{N}_{[0, N-1]}: x_{j+1}=A_{j} x_{j}+B_{j} u_{j}$

$\forall j \in \mathbb{N}_{[0, N-1]}: u_{j}=\left\{\begin{array}{l}u_{0}, j=0 \\ u_{j-1}+\Delta_{j-1}, \quad j \geq 1,\end{array}\right.$

$\forall j \in \mathbb{N}_{[1, N]}: F_{j} x_{j} \leq f_{j}$,

$\forall j \in \mathbb{N}_{[0, N-1]}: E_{j} u_{j} \leq e_{j}$,

$\forall j \in \mathbb{N}_{[0, N-2]}:\left\{\begin{array}{l}\Delta_{j} \leq \Gamma \delta_{j} \mathbf{1}, \\ \Delta_{j} \geq-\gamma \delta_{j} \mathbf{1}, \\ \Delta_{j} \leq c_{j}+\gamma\left(1-\delta_{j}\right) \mathbf{1}, \\ \Delta_{j} \geq c_{j}-\Gamma\left(1-\delta_{j}\right) \mathbf{1},\end{array}\right.$

$\forall j \in \mathbb{N}_{[0, N-2]}:-\gamma \mathbf{1} \leq c_{j} \leq \Gamma \mathbf{1}$,

$\forall j \in \mathbb{N}_{[0, N-2]}: \delta_{j} \in\{0,1\}$,

where $\gamma$ and $\Gamma$ are two large positive constants satisfying

$\gamma, \Gamma>\max _{j \in \mathbb{N}_{[0, N-2]}} \max _{u \in \mathbb{U}_{j}, v \in \mathbb{U}_{j+1}}\|u-v\|_{\infty}$.
Proof. By introducing new variables $c_{j} \in \mathbb{R}^{n_{u}}$ and $0-1$ variables $\delta_{j}, j \in \mathbb{N}_{[k+2, N]}$, we define $\Delta_{j}=\delta_{j} c_{j}$, i.e., $\Delta_{j}=0$ if $\delta_{j}=0$ and $\Delta_{j}=c_{j}$ if $\delta_{j}=1$. And it follows that $\forall j \in \mathbb{N}_{[k+1, N]}$ :

$\Delta_{j}=\delta_{j} c_{j} \Leftrightarrow\left\{\begin{array}{l}\Delta_{j} \leq \delta_{j} \Gamma \mathbf{1}, \\ \Delta_{j} \geq \delta_{j} \gamma \mathbf{1}, \\ \Delta_{j} \leq c_{j}+\left(1-\delta_{j}\right) \gamma \mathbf{1}, \\ \Delta_{j} \geq c_{j}-\left(1-\delta_{j}\right) \Gamma \mathbf{1}, \\ -\gamma \mathbf{1} \leq c_{j} \leq \Gamma \mathbf{1},\end{array}\right.$

where $\Gamma$ and $\gamma$ are two positive numbers satisfying (13). Then, we get the optimization problem $\mathcal{P}_{[0, N]}^{4}\left(x_{0}\right)$.

Remark 4.6. The statements in Remark 4.5 also apply with $\gamma$ and $\Gamma$ in (13). In addition, the optimization problem $\mathcal{P}_{[0, N]}^{4}\left(x_{0}\right)$ is equivalent to $\mathcal{P}_{[0, N]}^{2}\left(x_{0}\right)$, while the optimal solution to $\mathcal{P}_{\left[k_{i}, N\right]}^{3}\left(x_{k_{i}}\right)$ is a suboptimal solution to $\mathcal{P}_{\left[k_{i}, N\right]}^{1}\left(x_{k_{i}}\right)$.

\section{Examples}

This section provides three examples to illustrate the effectiveness of our proposed algorithms. The following numerical experiments were run in Matlab R2016a with MPT toolbox (Herceg et al., 2013) on a Dell laptop with Window 7, Intel i7-6600U CPU $2.80 \mathrm{GHz}$ and $16.0 \mathrm{~GB}$ RAM.

Example 1. Compare the proposed robust self-triggered algorithm with the robust self-triggered MPC in Brunner et al. (2014). Consider the same model as in Brunner et al. (2014), where $A=$ $[11 ; 01], B=[0.50]^{T}$. The constraint sets are $\mathbb{X}=\left\{z \in \mathbb{R}^{2}\right.$ $\left.[-20-8]^{T} \leq z \leq\left[\begin{array}{ll}20 & 8\end{array}\right]^{T}\right\}, \mathbb{U}=\{z \in \mathbb{R} \mid-4.5 \leq z \leq 4.5\}, \mathbb{W}=$ $\left\{z \in \mathbb{R}^{2} \mid[-0.25-0.25]^{T} \leq z \leq\left[\begin{array}{ll}0.25 & 0.25\end{array}\right]^{T}\right\}$. The initial state is $\left[\begin{array}{ll}10 & 6\end{array}\right]^{T}$.

Let $Q=\left[\begin{array}{lll}1 & 0 ; 0 & 1\end{array}\right]$ and $R=0.1$ be the weight matrices in the objective function. By solving the discrete-time algebraic Riccati equation, we obtain the matrix $P=[2.05990 .5916 ; 0.5916$ 1.4228] and the corresponding optimal feedback gain $K=$ $[-0.6167-1.2703]$. The control objective is to steer the state to the robust invariant set, denoted by $\Omega$ (the red region in Fig. 1), which is computed by the method in Mayne, Seron, and Raković (2005). The implementation will stop if the state enters the robust invariant set.

For the robust self-triggered control in this paper, the terminal set of the target tube is $\Omega$. For the robust self-triggered MPC in Brunner et al. (2014), we choose the maximal inter-sampling time $M_{\max }=4$. The state trajectories for 100 realizations of the uncertainty sequence are depicted in Fig. 1. We compare the two different methods for several indexes, of which the average is taken over 500 realizations.

- Average inter-sampling time: The average inter-sampling time is $\bar{M}=1.2045$ under the robust self-triggered MPC of Brunner et al. (2014) while it is $\bar{M}=1.3333$ under the self-triggered scheme of this paper. Thus, our control scheme achieves an average communication reduction by 9.66\% more than that of the self-triggered MPC.

- Average online computation time: The average online computation time at each sampling instant is $0.5758 \mathrm{~s}$ for the robust self-triggered MPC while it is $0.1964 \mathrm{~s}$ for our control method despite the presence of integer variables.

- Average performance: The performance measure is defined by $J=\sum_{k=0}^{T_{\text {run }}}\left(\left\|x_{k}\right\|_{Q}^{2}+\left\|u_{k}\right\|_{R}^{2}\right)$ where $T_{\text {run }}$ is the time when the state enters the robust invariant set. The robust selftriggered MPC achieves a slightly better average performance than our scheme. The performance measure is 591.9191 for MPC while it is 621.4552 for our scheme. 


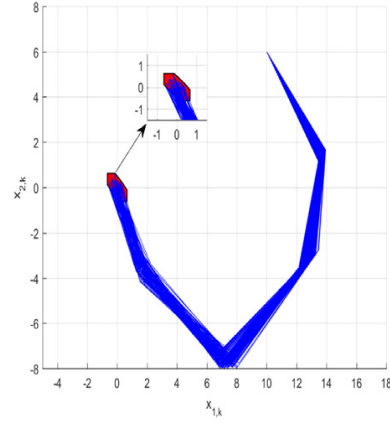

(a)

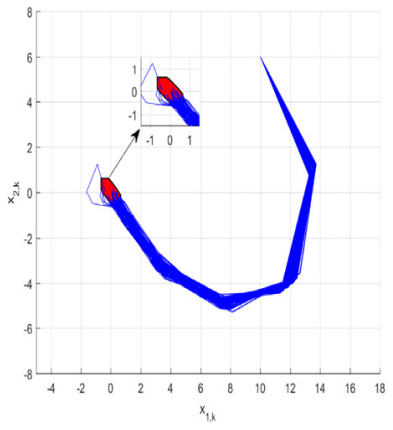

(b)
Fig. 1. State trajectories under Algorithm 1 and robust self-triggered MPC (Brunner et al., 2014) for 100 realizations of the uncertainty sequence. (a) Algorithm 1; (b) Robust self-triggered MPC. The algorithms terminate when the state enters the robust invariant set (the red region). (For interpretation of the references to color in this figure legend, the reader is referred to the web version of this article.)

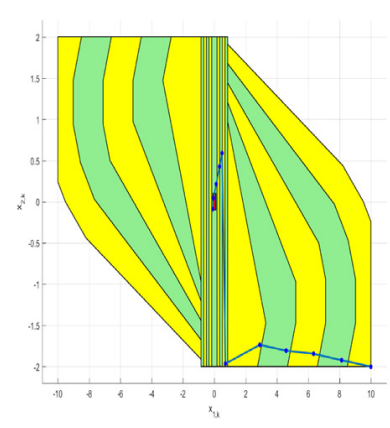

(a) State

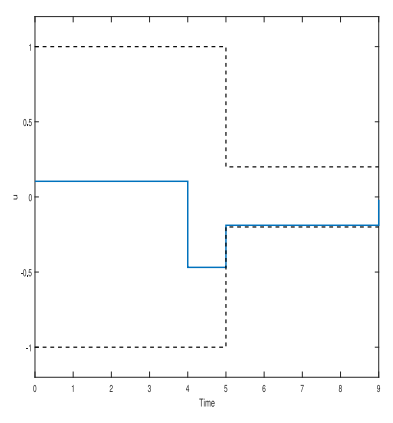

(b) Control input
Fig. 2. State trajectory and control input trajectory under Algorithm 1 for one realization of the uncertainty sequence. (For interpretation of the references to color in this figure legend, the reader is referred to the web version of this article.)

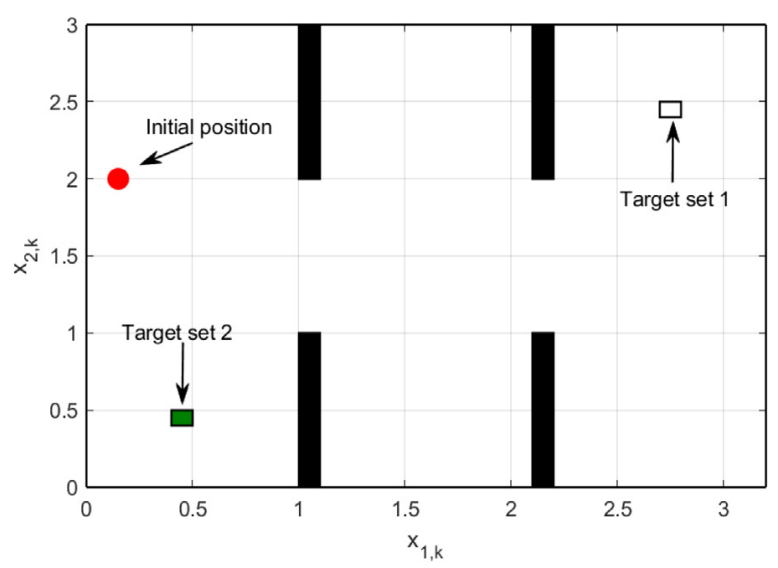

Fig. 3. Scenario.

Example 2. Consider a time-varying linear system (5), where $A_{k}=[11 ; 01], \quad B_{k}=[1 ; 0.5], \quad k \in \mathbb{N}_{[0,5]}$ and $A_{k}=$ $[10 ; 10], B_{k}=[1 ; 0.5], k \in \mathbb{N}_{[6,10]}$. Note that $A_{k}, k \in \mathbb{N}_{[6,10]}$, are singular. Let $N=10$. The input constraints are $\left|u_{k}\right| \leq 1, k \in \mathbb{N}_{[0,5]}$, and $\left|u_{k}\right| \leq 0.2, k \in \mathbb{N}_{[6,9]}$. The terminal constraint set is $\mathbb{X}_{N}=\{z \in$ $\left.\mathbb{R}^{2} \mid[-0.01 ;-0.01] \leq z \leq[0.01 ; 0.01]\right\}$. And the state constraint set is $\mathbb{X}_{k}=\left\{z \in \mathbb{R}^{2} \mid[-10 ;-2] \leq z \leq[10 ; 2]\right\}, k \in \mathbb{N}_{[0,9]}$. The disturbance set is $\mathbb{W}_{k}=\left\{z \in \mathbb{R}^{2} \mid[-0.03 ;-0.03] \leq z \leq\right.$ [0.03; 0.03]\}, $k \in \mathbb{N}_{[0,10]}$.
Algorithm 1 is implemented for the system with the initial state $[10-2]^{T}$. Fig. 2 depicts the state trajectory and the control input trajectory under Algorithm 1 for one realization of the disturbance sequence. In subfigure (a), the red square is the target set and the yellow and light green regions are the sets $\mathbb{X}_{k}^{*}, k \in$

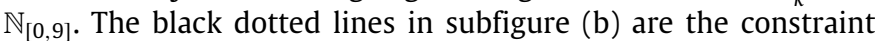
bounds on the control input. From subfigure (b), 3 updates for control inputs are needed to tolerate the disturbances as well as guarantee the constraints satisfaction.

Example 3. Consider a mobile robot with dynamics (5), where $A_{k}=\left[\begin{array}{lll}1 & 0 ; 0 & 1\end{array}\right], \quad B_{k}=[0.10 ; 00.1], \quad \forall k \in \mathbb{N}$. The input constraint set is $\mathbb{U}_{k}=\left\{z \in \mathbb{R}^{2} \mid\left[\begin{array}{ll}-0.6 & -0.6\end{array}\right]^{T} \leq z \leq\left[\begin{array}{ll}0.6 & 0.6\end{array}\right]^{T}\right\}$, $k \in \mathbb{N}$. The robot moves in a closed workspace, as shown in Fig. 3, in which there are some obstacles (the black rectangles). The robot should achieve collision avoidance with the obstacles and the boundaries of the workspace. We set the safe distance as 0.1. In addition, the robot can exchange the information (the position and the control input) with the control center via a bandwidthlimited communication network. At each time, the robot can only receive one control input from the control center. The initial position is $\left[\begin{array}{ll}0.15 & 2\end{array}\right]^{T}$. The target set 1 is $\left\{z \in \mathbb{R}^{2} \mid\left[\begin{array}{ll}2.7 & 2.4\end{array}\right]^{T} \leq\right.$

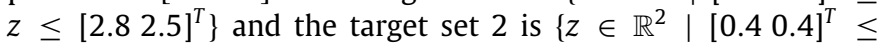
$\left.z \leq\left[\begin{array}{ll}0.5 & 0.5\end{array}\right]^{T}\right\}$. A sequence of temporal constrained tasks for the mobile robot are

- stage 1: the robot should arrive at the target set 1 before $k=60$;

- stage 2: the robot should stay in target set 1 for at least 10 time steps after arrival.

- stage 3: the robot should arrive at the target set 2 before $k=140$.

To save the communication recourses, our self-triggered control strategies are implemented. We choose the convex inner approximations of the backward reachable sets (which are the intersection between the predecessor sets and the safe regions). As mentioned in Remark 3.1, these approximations still respect the feasibility and the constraint satisfactions.

In the first case, assume that the disturbance set is $\mathbb{W}_{k}=\{z \in$ $\left.\mathbb{R}^{2} \mid[-0.01-0.01]^{T} \leq z \leq[0.010 .01]^{T}\right\}$. Subfigures (a)-(b) of Fig. 4 depict the state trajectories for stages 1 and 3 under Algorithm 1 . The yellow and light green regions are the sets $\mathbb{X}_{k}^{*}$. The control input trajectory is shown in subfigure (c) of Fig. 4 with the times of control update being 15 .

In the second case, assume that $\mathbb{W}_{k}=\left[\begin{array}{ll}0 & 0\end{array}\right]^{T}, \quad \forall k \in \mathbb{N}$. Subfigures (a)-(b) of Fig. 5 depict the state trajectories for stage 1 and 3 by using the control with minimum number of samplings. The yellow and light green regions are the convex approximations of the sets $\mathbb{X}_{k}^{*}$. The control input trajectory is shown in subfigure (c) of Fig. 5 with the times of control update being 6 .

\section{Conclusion}

In this paper, we proposed a robust self-triggered control algorithm for time-varying uncertain systems with constraints. By using reachability analysis, the constraint satisfactions and recursive feasibility were guaranteed. The proposed algorithm provided us a geometric interpretation for self-triggered control. The problem of control with minimum number of samplings was investigated for deterministic constrained systems. For linear systems with polyhedral constraints, the proposed methods were reformulated as computationally tractable MILP problems. In simulations, the results were compared with robust self-triggered MPC and applied to the robot motion planning. 


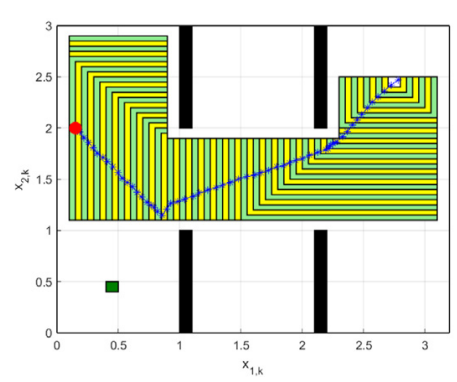

(a) State of stage 1

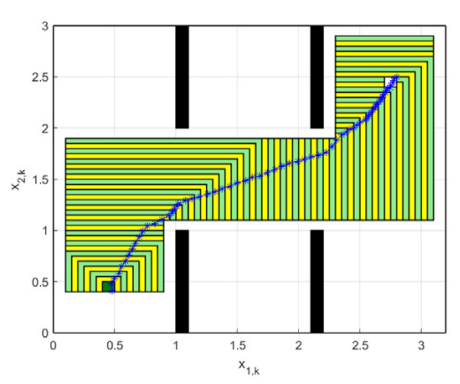

(b) State of stage 3
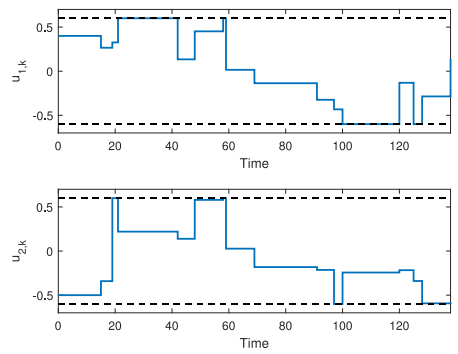

(c) Control input

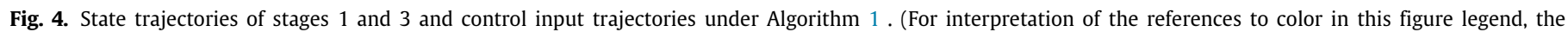
reader is referred to the web version of this article.)

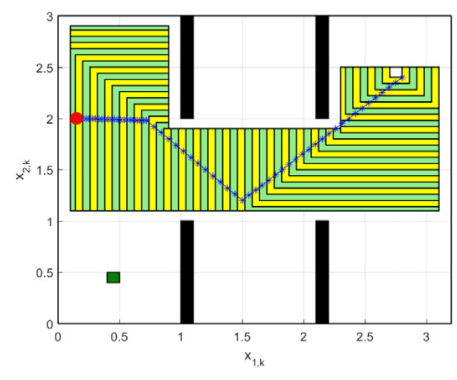

(a) State of stage 1

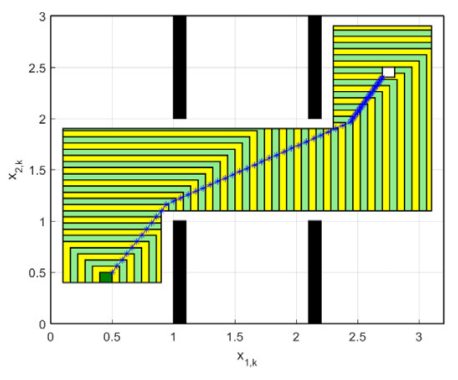

(b) State of stage 3
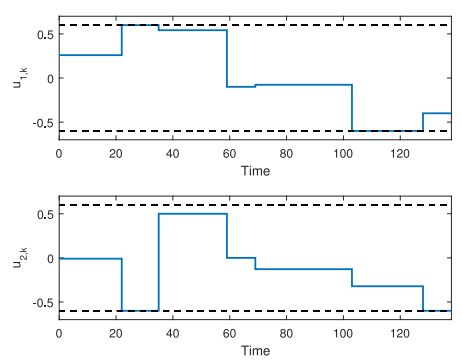

(c) Control input

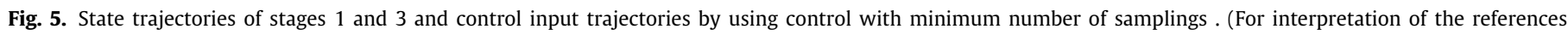
to color in this figure legend, the reader is referred to the web version of this article.)

\section{References}

AlKhatib, M., Girard, A., \& Dang, T. (2017). Self-triggered control for sampleddata systems using reachability analysis. In Proceedings of 20th IFAC world congress (pp. 7881-7886).

Althoff, M., \& Krogh, B. H. (2014). Reachability analysis of nonlinear differential-algebraic systems. IEEE Transactions on Automatic Control, 59(2), 371-383.

Barradas Berglind, J. D. J., Gommans, T. M. P., \& Heemels, W. P. M. H. (2012). Self-triggered MPC for constrained linear systems and quadratic costs. In Proceedings of IFAC conference on nonlinear model predictive control (pp. 342-348)

Bertsekas, D. P., \& Rhodes, I. B. (1971). On the minimax reachability of target sets and target tubes. Automatica, 7(2), 233-247.

Blanchini, F., \& Miani, S. (2007). Set-theoretic methods in control. Boston: Springer. Brunner, F. D., Heemels, W. P. M. H., \& Allgöwer, F. (2014). Robust selftriggered MPC for constrained linear systems. In Proceedings of European control conference (pp. 472-477).

Brunner, F. D., Heemels, W. P. M. H., \& Allgöwer, F. (2016). Robust self-triggered MPC for constrained linear systems: A tube-based approach. Automatica, 72, 73-83.

Chen, M., Herbert, S. L., Vashishtha, M., Bansal, S., \& Tomlin, C. J. (2018). Decomposition of reachable sets and tubes for a class of nonlinear systems. IEEE Transactions on Automatic Control, 63(11), 3675-3688.

Dai, L., Gao, Y. L., Xie, L. H., Johansson, K. H., \& Xia, Y. Q. (2018). Stochastic self-triggered model predictive control for linear systems with probabilistic constraints. Automatica, 92, 9-17.

Fleming, J., Kouvaritakis, B., \& Cannon, M. (2013). Regions of attraction and recursive feasibility in robust MPC. In Proceedings of the 21st mediterranean conference on control and automation (pp. 801-806).

Gutman, P. O., \& Hagander, P. (1985). A new design of constrained controllers for linear systems. IEEE Transactions on Automatic Control, 30(1), 22-33.

Hashimoto, K., Adachi, S., \& Dimarogonas, D. V. (2018). Aperiodic sampled-data control via explicit transmission mapping: a set invariance approach. IEEE Transactions on Automatic Control, 63(10), 3523-3530.
Heemels, W. P. M. H., Johansson, K. H., \& Tabuada, P. (2012). An introduction to event-triggered and self-triggered control. In Proceedings of IEEE conference on decision and control (pp. 3270-3285).

Henriksson, E., Quevedo, D. E., Sandberg, H., \& Johansson, K. H. (2012). Selftriggered model predictive control for network scheduling and control. In Proceedings of IFAC symposium on advanced control of chemical processes ( $\mathrm{pp}$. 432-438).

Herceg, M., Kvasnica, M., Jones, C. N., \& Morari, M. (2013). Multi-parametric toolbox 3.0. In Proceedings of European control conference (pp. 502-510).

Lehmann, D., Kiener, G. A., \& Johansson, K. H. (2012). Event-triggered PI control: Saturating actuators and anti-windup compensation. In Proceedings of IEEE conference on decision and control (pp. 6566-6571).

Linderoth, J. T., \& Lodi, A. (2010). MILP software. Wiley Encyclopedia of Operations Research and Management Science.

Lygeros, J., Tomlin, C., \& Sastry, S. (1999). Controllers for reachability specifications for hybrid systems. Automatica, 35(3), 349-370.

Mayne, D. Q., Rawlings, J. B., Rao, C. V., \& Scokaert, P. O. (2000). Constrained model predictive control: Stability and optimality. Automatica, 36(6), 789-814.

Mayne, D. Q., Seron, M. M., \& Raković, . S. V. (2005). Robust model predictive control of constrained linear systems with bounded disturbances. Automatica, 41(2), 219-224.

Megiddo, N. (1984). Linear programming in linear time when the dimension is fixed. Journal of the ACM, 31(1), 114-127.

Mitchell, I. M. (2011). Scalable calculation of reach sets and tubes for nonlinear systems with terminal integrators: a mixed implicit explicit formulation. In Proceedings of the 14th ACM international conference on hybrid systems: Computation and control (pp. 103-112).

Raković, S. V., Kerrigan, E. C., Mayne, D. Q., \& Lygeros, J. (2006). Reachability analysis of discrete-time systems with disturbances. IEEE Transactions on Automatic Control, 51(4), 546-561.

Seuret, A., Prieur, C., Tarbouriech, S., \& Zaccarian, L. (2013). Event-triggered control with LQ optimality guarantees for saturated linear systems. In Proceedings of IFAC symposium on nonlinear control systems (pp. 341-346). 
Wu, W., Reimann, S., \& Liu, S. (2014). Event-triggered control for linear systems subject to actuator saturation. In Proceedings of IFAC world congress (pp. 9492-9497).

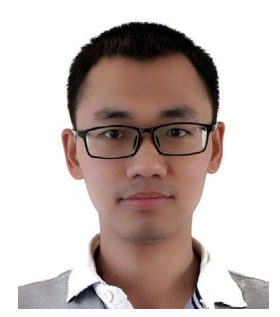

Yulong Gao received the B.S. degree in Automation in 2013 and the M.S. degree in Control Science and Engineering in 2016 from Beijing Institute of Technology, Beijing, China. Now he is a Ph.D. student at the School of Electrical Engineering and Computer Science, KTH Royal Institute of Technology. His main research interest includes stochastic control, model predictive control, and event-triggered control with application of transportation systems and multi-agent systems.

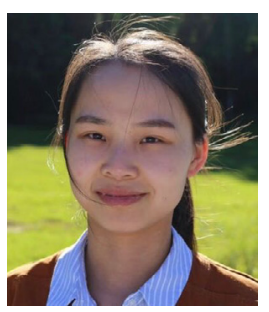

Pian Yu received the M.Sc. degree in Control Science and Engineering in 2016 from Wuhan University. Currently, she is a Ph.D. student at the Division of Decision and Control Systems, School of Electrical Engineering and Computer Science, KTH Royal Institute of Technology. Her main research interest includes multi-agent systems, event-triggered communication and control, dynamic scheduling and formal methods.

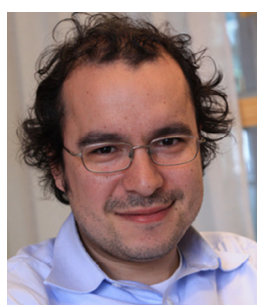

Dimos V. Dimarogonas received the Diploma in Electrical and Computer Engineering in 2001 and the Ph.D. in Mechanical Engineering in 2007, both from the National Technical University of Athens (NTUA), Greece. From May 2007 to February 2009, he was a Postdoctoral Researcher at the Automatic Control Laboratory, School of Electrical Engineering, KTH Royal Institute of Technology, Stockholm, Sweden, and a Postdoctoral Associate at the Laboratory for Information and Decision Systems, Massachusetts Institute of Technology (MIT) Cambridge, MA, USA. He is currently a Professor in Division of Decision and Control Systems, School of Electrical Engineering and Computer Science, KTH Royal Institute of Technology.

His current research interests include multi-agent systems, hybrid systems, robot navigation, networked control and event-triggered control. Dr. Dimarogonas was awarded a Docent in Automatic Control from KTH in 2012. He serves on the Editorial Board of Automatica, the IEEE Transactions on Automation Science and Engineering and the IET Control Theory and Applications, and is a member of the Technical Chamber of Greece.

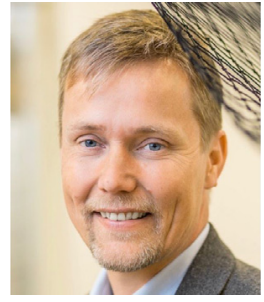

Karl Henrik Johansson received the M.Sc. and Ph.D. degrees in electrical engineering from Lund University, Lund, Sweden. He is the Director of the Stockholm Strategic Research Area ICT The Next Generation and a Professor with the School of Electrical Engineering and Computer Science, KTH Royal Institute of Technology. He has held visiting positions with the University of California, Berkeley, California Institute of Technology, Nanyang Technological University, HKUST Institute of Advanced Studies, and Norwegian University of Science and Technology.

His research interests include networked control systems, cyber-physical systems, and applications in transportation, energy, and automation. He is a member of the IEEE Control Systems Society Board of Governors, the IFAC Executive Board, and the European Control Association Council. He has received several best paper awards and other distinctions. He has been awarded Distinguished Professor with the Swedish Research Council and Wallenberg Scholar. $\mathrm{He}$ is a recipient of the Future Research Leader Award from the Swedish Foundation for Strategic Research and the triennial Young Author Prize from IFAC. He is a Fellow of the Royal Swedish Academy of Engineering Sciences. He is an IEEE Distinguished Lecturer.

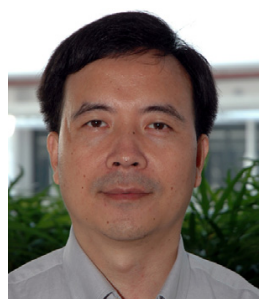

Lihua Xie received the B.E. and M.E. degrees in electrical engineering from Nanjing University of Science and Technology in 1983 and 1986, respectively, and the Ph.D. degree in electrical engineering from the University of Newcastle, Australia, in 1992. Since 1992, he has been with the School of Electrical and Electronic Engineering, Nanyang Technological University, Singapore, where he is currently a professor and Director, Delta-NTU Corporate Laboratory for Cyber-Physical Systems. He served as the Head of Division of Control and Instrumentation from July 2011 to June 2014. He held teaching appointments in the Department of Automatic Control, Nanjing University of Science and Technology from 1986 to 1989.

Dr. Xie's research interests include robust control and estimation, networked control systems, multi-agent networks, localization and unmanned systems. He is an Editor-in-Chief for Unmanned Systems and an Associate Editor for IEEE Transactions on Network Control Systems. He has served as an editor of IET Book Series in Control and an Associate Editor of a number of journals including IEEE Transactions on Automatic Control, Automatica, IEEE Transactions on Control Systems Technology, and IEEE Transactions on Circuits and Systems-II. He is an elected member of Board of Governors, IEEE Control System Society (Jan 2016-Dec 2018). Dr. Xie is a Fellow of IEEE and Fellow of IFAC. 